\title{
The optimal feedbacks in the mathematical model of chemotherapy for a nonmonotonic therapy function
}

\author{
N. Novoselova \\ Krasovskii Institute of Mathematics and Mechanics, Ural Branch of the RAS, Yekaterinburg, Russia \\ Ural Federal University, Yekaterinburg, Russia \\ e-mail:n.g.novoselova@gmail.com
}

Key words: therapy function, optimal control, optimal feedbacks, Hamilton-Jacobi-Bellman equation, Cauchy method of characteristics, Rankin-Hugoniot line

Motivation and Aim: We investigate a pharmacokinetic problem for a deterministic nonlinear system with piecewise monotonic dynamics describing the process of chemotherapy of a malignant tumor. We consider the case when the therapy function, which describes the effect of the drug on the cell growth rate, has two maxima.

Methods and Algorithms: The work presents results of numerical calculation of the optimal result (the value function) and optimal positional strategy of therapy (optimal feedbacks) in a corresponding optimal control problem. The construction use the fact that the value function is the unique minimax (viscosity) solution $[1,2]$ of the Cauchy problem for the basic Hamilton-Jacobi-Bellman (HJB) equation. By means of the continuous gluing of a finite number of smooth functions obtained by the Cauchy method of characteristics for auxiliary linear HJB equations, the continuous function $\phi$ is constructed. The paper [3] proves that the constructed function $\phi$ coincides with the value function. Results: A new element of the construction is the construction of a line of nonsmooth gluing using the Rankin-Hugoniot conditions [4,5]. This line plays a key role for the optimal feedback strategy, because it determines its discontinuity line. The results of numerical calculations of the Rankin-Hugoniot line are exposed. Comparison with the results for the case of a single maximum in the therapy function in this model [6] is given. Acknowledgements: Supported by the RFBR (No. 17-01-00074).

\section{References}

1. Crandall M.G., Evans L.C., Lions P.-L. (1984) Some properties of viscosity solutions of Hamilton-Jacobi equations, Trans. Amer. Math. Soc. 282(2):487-502.

2. Subbotin A.I. (1995) Generalized Solutions of First-Order PDEs: The Dynamical Optimization Perspective. Birkhauser: Boston.

3. Subbotina N.N., Novoselova N.G. (2017) Optimal result in a control problem with piecewise monotone dynamics. Journal "Proceedings of the Institute of Mathematics and Mechanics". 23(4):265-280.

4. Goritskiy A.Yu., Kruzhkov S.N., Chechkin G.A. (1999) First-Order Partial Differential Equations (Study Guide). Moscow: Lomonosov Moscow State University, Faculty of Mechanics and Mathematics.

5. Subbotina N.N., Kolpakova E.A., Tokmantsev T.B., Shagalova L.G. (2013) The Method of Characteristics for Hamilton Jacobi Bellman equations. RIO UrO RAN, Yekaterinburg.

6. Chumerina E.S. (2009) Choice of optimal strategy of tumor chemotherapy in Gompertz model. Journal Computer Systems Sciences International. 48(2):325-331. 